\title{
Identification et caractérisation de la dynamique de la grande faune dans le baï de Momba (nord-est Gabon)
}

\author{
Fred Loïque MINDONGA-NGUELET ${ }^{1,2}$, Christophe Roland ZINGA KOUMBA ${ }^{1 *}$, \\ Donald MIDOKO IPONGA ${ }^{1}$, Ephrem NZENGUE ${ }^{1,2}$, Clency O. MIKALA ${ }^{2}$, \\ Joseph OKOUYI OKOUYI ${ }^{1,3}$, Bertrand M'BACHI ${ }^{2}$, Jacques François MAVOUNGOU ${ }^{1,2}$ \\ et Mutambwe $\mathrm{SHONGO}^{4}$
}

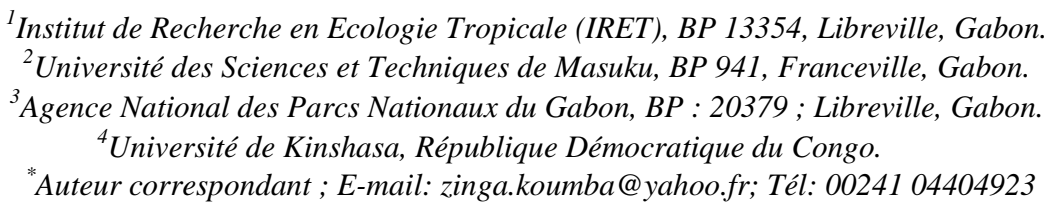

\section{REMERCIEMENTS}

Nous tenons à remercier l'appui financier du projet CoForSet qui a obtenu un financement de la FRB, à travers l'appel à projets 2013 "Scénarios de la biodiversité en Afrique subsaharienne".

\section{RESUME}

Les baïs sont des clairières marécageuses localisées généralement au cœur des forêts du Bassin du Congo. Ce sont des écosystèmes particulièrement riches en espèces animales et végétales. Si les connaissances portant sur l'origine de ces milieux sont bien documentées, les espèces de la faune sauvage (éléphants, buffles, sitatungas, etc.) colonisant ces écosystèmes demeurent encore mal connues. Par ailleurs, les informations qui existent sur ces espèces fauniques restent fragmentaires. C'est pour ces raisons qu'une étude basée sur les méthodes d'observation de la faune (scan sampling et animal focal) a été conduite dans le baï de Momba durant 33 jours. Ce travail visait l'identification de la macrofaune présente dans ce type de milieux et l'analyse de la dynamique de cette faune. Au total, 969 animaux ont été observés. Ces animaux ont été représentés majoritairement par les sitatungas (Tragelaphus spekei), les éléphants (Loxodonta africana cyclotis), les buffles (Syncerus caffer nanus) et les colobes (Colobus guereza). En termes de fréquence d'observation, les sitatungas (27\%) ont été le groupe le plus abondant, suivis par les éléphants (8\%) et les buffles (7\%). Les autres animaux ont été faiblement observés avec une fréquence de moins de $2 \%$. La fréquentation du baï de Momba par ces espèces témoigne du rôle crucial que pourrait jouer les zones humides dans la gestion durable de la biodiversité dans le Bassin du Congo. Aussi, il apparaît nécessaire que des mesures de protection et de suivi de la dynamique de ces peuplements soient rapidement entreprises afin de protéger la biodiversité faunique de ce milieu.

(C) 2016 International Formulae Group. All rights reserved.

Mots clés : Loxodonta africana cyclotis, Syncerus caffer nanus, Tragelaphus spekei,Colobus guereza, baï de Momba, Gabon. 


\title{
Identification and specification of the macrofauna dynamics in the Momba baï (North-East Gabon)
}

\begin{abstract}
The Baïs (swampy clearings) are generally localized in the heart of the forests in most of the Congo Basin areas. They are particularly rich ecosystems in terms of animal and plant species. If knowledge of the origin of these specific environments are well documented, species of wild animals (elephants, buffalo, sitatunga, etc.) colonizing these ecosystems are still poorly known. Moreover, the available information that may exist on these wildlife species remain fragmented. The aim of this work was to identify the macrofauna in this type of ecosystem and analyze the dynamics of this fauna. The study was based on direct wildlife observation methods (scan sampling and focal animal) in the baï of Momba for 33 days. A total of 969 animals were observed. These animals were mainly represented by sitatunga (Tragelaphus spekei), the elephants (Loxodonta africana cyclotis), the buffalos (Syncerus caffer nanus) and colobus (Colobus guereza). In terms of observation frequencies, sitatungas $(27 \%)$ were the most abundant group visiting the Baï, followed by elephants (8\%) and buffalo (7\%). Other animals were weakly observed with a frequency of less than $2 \%$. The presence of these wildlife species in Momba Baï, may demonstrate the crucial role that wetland could play in the sustainable management of biodiversity and specially wildlife in the Congo Basin forest. Then, for conservation purposes, it appears necessary to monitor the dynamic of these species and develop conservation measures to protect wildlife biodiversity of this environment.
\end{abstract}

(C) 2016 International Formulae Group. All rights reserved.

Keywords: Loxodonta africana cyclotis, Syncerus caffer nanus, Tragelaphus spekei, Colobus guereza, Momba baï, Gabon.

\section{INTRODUCTION}

Les clairières forestières encore appelées baï sont des zones humides riches en espèces animales et végétales que l'on retrouve dans les forêts du Bassin du Congo (Vanleeuwe et al., 1998 ; Vande Weghe, 2006). Ce sont des écosystèmes particuliers dont les influences sur la concentration de la population animale sont encore mal connues malgré les observations et affirmations empiriques qui semblent confirmer un certain effet (Noupa et Nkongmeneck, 2008). Par ailleurs, ces milieux sont des pôles importants de concentration animale pour les grands herbivores tels que les éléphants (Loxodonta africana cyclotis), les buffles (Syncerus caffer nanus), les gorilles (Gorilla gorilla), les potamochères (Potamochoerus porcus) qui y trouvent une végétation digestible à croissance rapide, disponible tout le long de l'année (Nganongo, 1999, 2000). De plus, la richesse des baïs en sels minéraux (sodium, calcium, magnésium, etc.) (Magliocca et GauttierHion, 2011) et en eaux constitue pour ces animaux une source de complément alimentaire (Magliocca et al., 1999). Aussi, plusieurs études ont montré l'importance de ces clairières dans le maintien de la biodiversité. En effet, ces biotopes sont caractérisés par une végétation herbacée dominée par la famille des graminées jouant le rôle de pâturage naturel (Noupa et Nkongmeneck, 2008 ; Boupoya, 2010).

$\mathrm{Au}$ Gabon, les connaissances globales sur la biodiversité des baï et la dynamique d'évolution de cette biodiversité demeurent encore fragmentaires. Pourtant, ces milieux ont une importance écologique et biologique majeure dans le maintien de la grande faune dans les zones humides et dans les aires protégées (Vande Weghe, 2006). Par ailleurs, la grande faune présente dans des clairières forestières du Gabon reste jusqu'à présent assez mal connue. Il existe peu de données scientifiques qui caractérisent les interactions faune-faune et faune-flore de ces milieux et qui évaluent leur importance socioéconomique pour les populations riveraines (Temgoua, 2006 ; Momont, 2007 ; WCS, 2008). De même, la valorisation des clairières 
au Gabon en termes d'écotourisme passe nécessairement par la connaissance des animaux présents dans ces milieux. Aussi cette connaissance est-elle utile dans l'élaboration des stratégies de gestion durable de la faune et des zones humides.

Pour recueillir les données sur la grande faune fréquentant les clairières forestières au Gabon, une étude sur l'inventaire de la macrofaune a été conduite dans le baï de Momba durant la grande saison sèche. L'objectif de ce travail était d'identifier les espèces de la grande faune fréquentant cette clairière et de caractériser leur dynamique.

\section{MATERIEL ET METHODES Zone d'étude}

La présente étude a été réalisée dans le baï de Momba située au Nord-Est du Gabon, à environ 160 kilomètres de la ville de Makokou (Figure 1).

Le climat de cette région est de type équatorial, caractérisé par la double alternance de saisons sèches (de mi-décembre à mi-mars, puis de mi-juin à mi-septembre) et de saisons des pluies (mi-mars à mi-juin puis miseptembre à mi-décembre) (Vande, 2006). La température moyenne est de $24{ }^{\circ} \mathrm{C}$ avec un minimum de $21,7{ }^{\circ} \mathrm{C}$ en juillet et un maximum de $25{ }^{\circ} \mathrm{C}$ en avril. Les amplitudes thermiques annuelles et journalières sont faibles (environ 3,3 ${ }^{\circ} \mathrm{C}$ ). La pluviométrie annuelle moyenne est de l'ordre de $1700 \mathrm{~mm}$ les plus fortes précipitations ayant lieu en octobre et novembre, puis en mars et mai (Vande, 2006). Le paysage est caractérisé par une végétation dominée par les Cyperaceae, les Poaceae et les Rubiaceae (Zinga et al., 2013a, 2013b, 2014). La grande faune est représentée par plusieurs espèces de mammifères dont les éléphants (Loxodonta africana cyclotis), les buffles (Syncerus caffer nanus) et les sitatungas (Tragelaphus spekei) (Zinga et al., 2014).

\section{Méthodes}

La méthode du scan sampling (Turkalo et Fay, 1995) et celle de l'animal focal ont été utilisées pour recueillir les informations sur la grande faune fréquentant le baï de Momba. La technique du scan sampling a consisté à compter, à intervalles de temps réguliers toutes les 30 minutes), le nombre d'espèces animales présentes dans le baï. Quant à la méthode de l'animal focal, elle a consisté à prendre d'autres informations telles que l'identité des éléphants fréquentant spécifiquement le baï de Momba et leur âge. La reconnaissance de ces éléphants s'est faite sur la base de leurs caractères morphologiques visibles : cicatrices sur le corps, blessures, nombre de défenses, trous sur les oreilles. Alors que, l'estimation de l'âge des éléphants s'est fondée sur la méthode proposée par Laws et al. (1975) et par Lee et Moss (1995).

Toutes les observations ont été faites au niveau d'un mirador de $8,5 \mathrm{~m}$ de haut et de $3 \mathrm{~m}$ de côté comprenant deux niveaux. Le premier niveau se trouve à $4 \mathrm{~m}$ du sol tandis que le second est à $6,5 \mathrm{~m}$. Cet imposant dispositif est situé à moins de $20 \mathrm{~m}$ de la plaine du Baï en lisière forestière. Par ailleurs, le matériel d'observation utilisé était constitué d'une longue vue à fort grossissement de marque Nikon, d'un appareil photo numérique de marque Nikon, pouvant faire des séquences vidéo et d'une paire de jumelle.

Les différentes observations ont été réalisées pendant trois mois, de juin à août 2011, c'est-à-dire durant la grande saison sèche. L'heure du début des observations a été fixée à 7 h 30 et celle de la fin à 17 h 30, soit dix (10) heures d'observation par jour.

\section{Analyse statistique}

Pour apprécier le niveau de fréquentation du baï par chaque espèce animale plusieurs paramètres ont été calculés à savoir :

- Le taux de fréquentation : $\%$ Fr $=\frac{\text { Nombre total } d \text { invidus d une espèce }}{\text { Nombre total d individus observés }} \times 100$

Avec: \% Fr le taux de fréquentation d'une espèce.

- Le taux de présence journalier:

$$
\% \mathrm{Pr}=\frac{\text { Nombre de jours de présence dune espèce }}{\text { Nombre de jours d'observation }} \times 100
$$

Avec: \% $\operatorname{Pr}$ le taux de présence journalière d'une espèce. 
- $\quad$ La moyenne quotidien d'observation: Moy obs $=\frac{\text { Nombre dindividus observés dune espèce }}{\text { Nombre de jours d'observation }} \times 100$ Avec: Moy obs la moyenne quotidienne d'observation.

\section{RESULTATS}

Fréquentation du baï par la faune observée Au total, 969 animaux ont été observés dans la clairière de Momba durant 330 heures. Ces animaux ont été répartis en plusieurs catégories : 7 espèces d'oiseaux, en 6 espèces de mammifères et en 1 espèce de reptiles. L'abondance et la fréquence spécifique de la diversité faunistique observée dans le baï de Momba sont consignées dans le tableau cidessous (Tableau 1).

Les résultats du Tableau 1 ont révélé que le baï de Momba est fortement fréquenté durant la saison sèche par plusieurs espèces animales dont les râles noirs (Limnocorax flavirostra.), les sitatungas (Tragelaphus spekei), les éléphants (Loxodonta africana cyclotis); les buffles (Syncerus caffer nanus), les colobes (Colobus guereza) et l'aigle pêcheur (Haliaeetus vocifer). Les autres espèces ont été très faiblement représentées, avec des taux de fréquentation inférieurs à $5 \%$.

Chez les oiseaux, le râle noir a été l'espèce la plus observée, avec un taux de fréquentation de $41 \%$. Les autres espèces ont été très faiblement observées avec $6 \%$ de taux de fréquentation. Chez les mammifères, les sitatungas ont été les espèces les mieux représentées, avec un taux de fréquentation de l'ordre de $29,5 \%$. Les éléphants et les buffles ont été faiblement observés avec environ $6 \%$ de taux de fréquentation. En ce qui concerne les reptiles, seul le varan (Varanus nititicus) a été observé.

Présence journalière de la grande faune dans le baï de Momba

Étant donné que la plus grande partie des espèces de grande faune a un taux de fréquentation inférieur à 5\%, seuls les sitatungas (Tragelaphus spekei), les éléphants (Loxodonta africana cyclotis) et les buffles (Syncerus caffer nanus) ont été pris en compte dans l'étude des grands mammifères fréquentant ce baï.

Les sitatungas ont été les mammifères les plus abondants (284 individus observés) et les plus fréquents dans le baï de Momba (Figure 2). En effet, les sitatungas (Tragelaphus spekei) ont été présents dans cette clairière durant toute la période de notre étude avec une moyenne journalière de 8 individus. De même, le pic de présence de cette espèce a été atteint entre le $30^{\text {ième }}$ et le $33^{\text {ième }}$ jour du suivi. Ce pic a été marqué par l'observation de 25 individus le $31^{\text {ième }}$ jour de l'étude.

L'analyse de la Figure 3 a indiqué que contrairement aux sitatungas, les éléphants ont été observés durant 18 jours, pendant toute la période d'étude avec un total de 76 individus vus. Cette espèce a présenté une fréquence d'apparition erratique pendant 3 à 4 jours ; celle-ci est marquée par une périodicité relativement stable de 2 à 3 jours. La moyenne journalière des individus observés pendant le suivi est relativement faible par rapport à celle des sitatungas (2 individus/jour).

Par ailleurs, les résultats du Tableau 2 révèlent que sur les 76 individus observés, 15 ont été identifiés de manière spécifique dont 9 femelles et 6 mâles. De plus, certains de ces individus, tels que Zowa, Etsitsi, Mossi et Rosia, ont été reconnus au moins deux fois dans ce milieu. Par contre, d'autres individus n'ont été observés qu'une seule fois durant toute la période d'étude. De manière générale, plus de la moitié des éléphants identifiés avait un âge supérieur à 45 ans.

En ce qui concerne les buffles, ils ont représenté le troisième groupe taxonomique le plus observé après les sitatungas et les éléphants. Sur les 33 jours de suivi, ces animaux ont été présents pendant 17 jours dans ce baï avec un total de 64 individus observés. La moyenne journalière d'observation a été de 1 individu/jour, avec une fréquence d'apparition allant de 1 à 8 jours successifs (Figure 4). La périodicité de présence de ces animaux dans le baï a été hétérogène allant de 1 à 5 jours d'absence. 


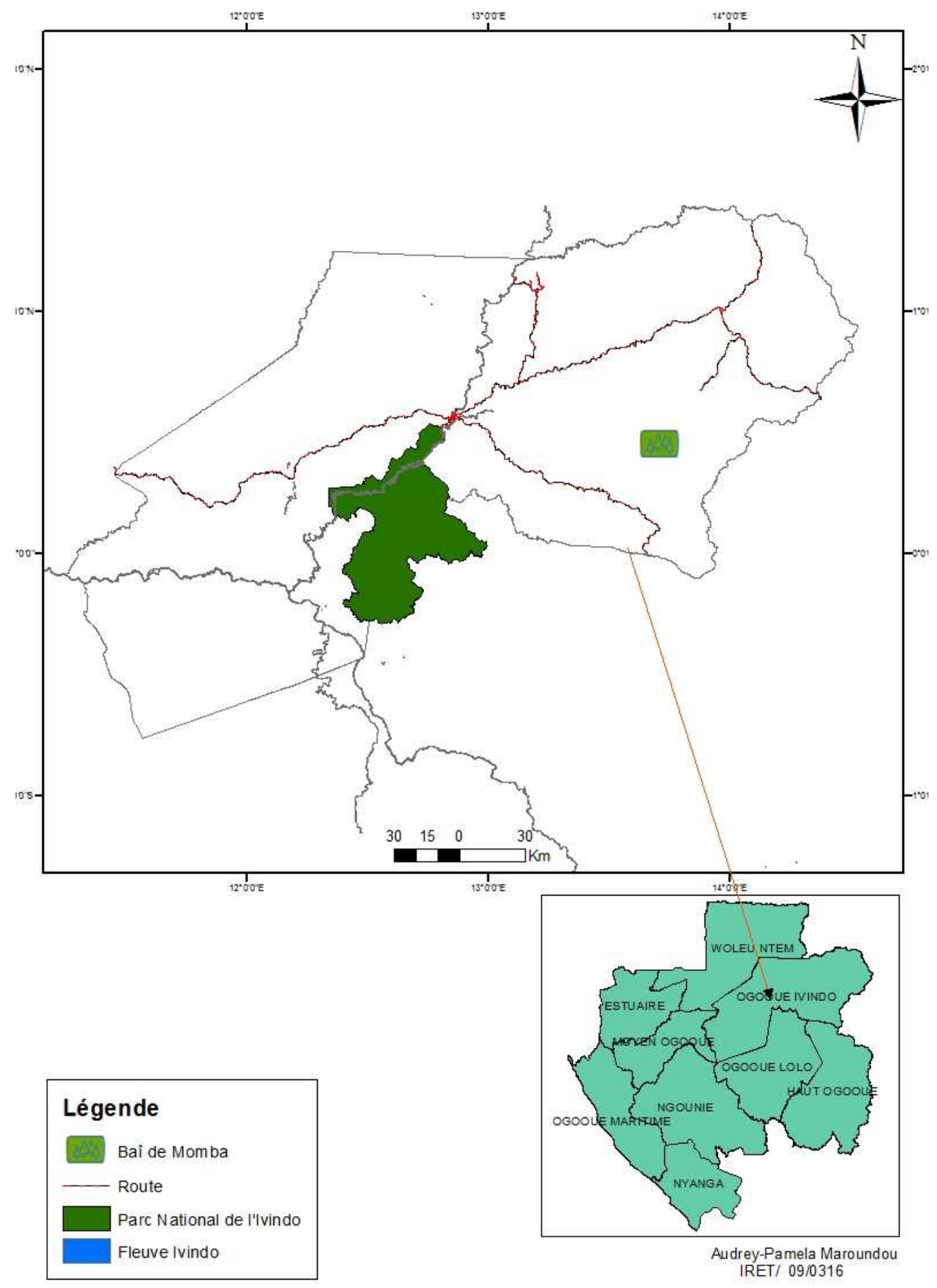

Figure 1 : Localisation du baï de Momba. 


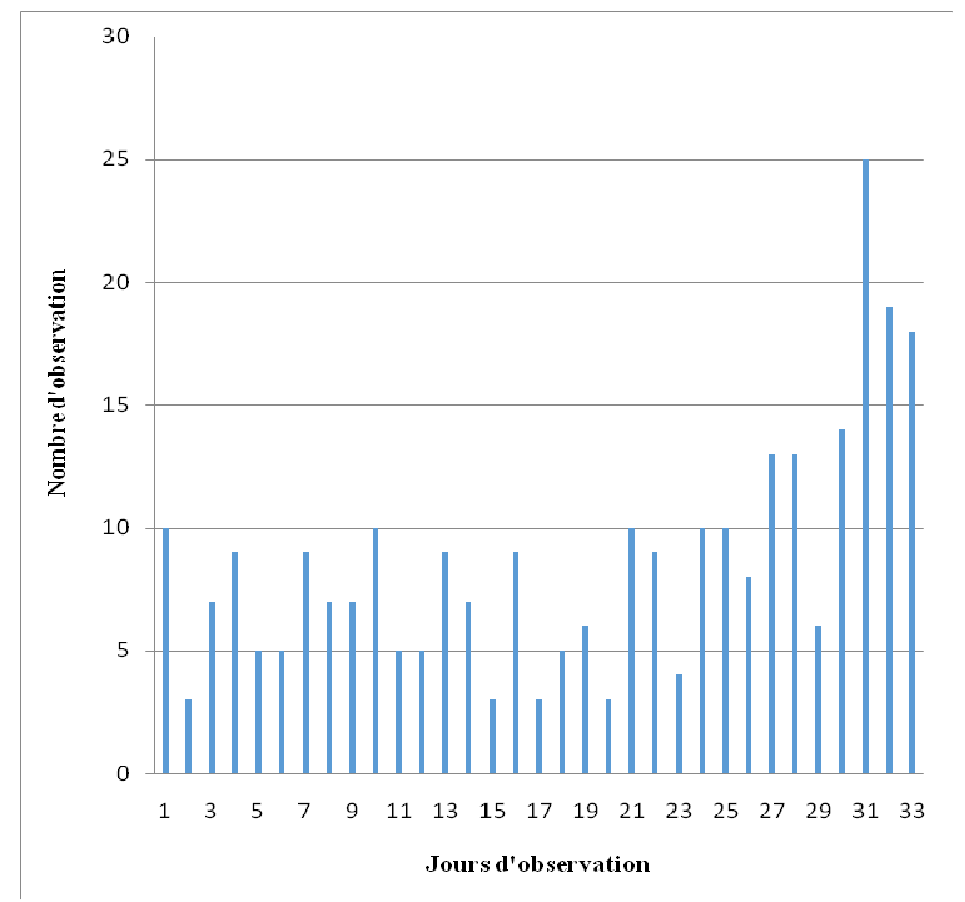

Figure 2 : Répartition des sitatungas observés dans le baï durant toute la période de l'étude. L'axe des $\mathrm{X}$ correspond au nombre de jours d'observation et l'axe des $\mathrm{Y}$ au nombre d'apparitions des buffles transformé en log (effectif observé+1).

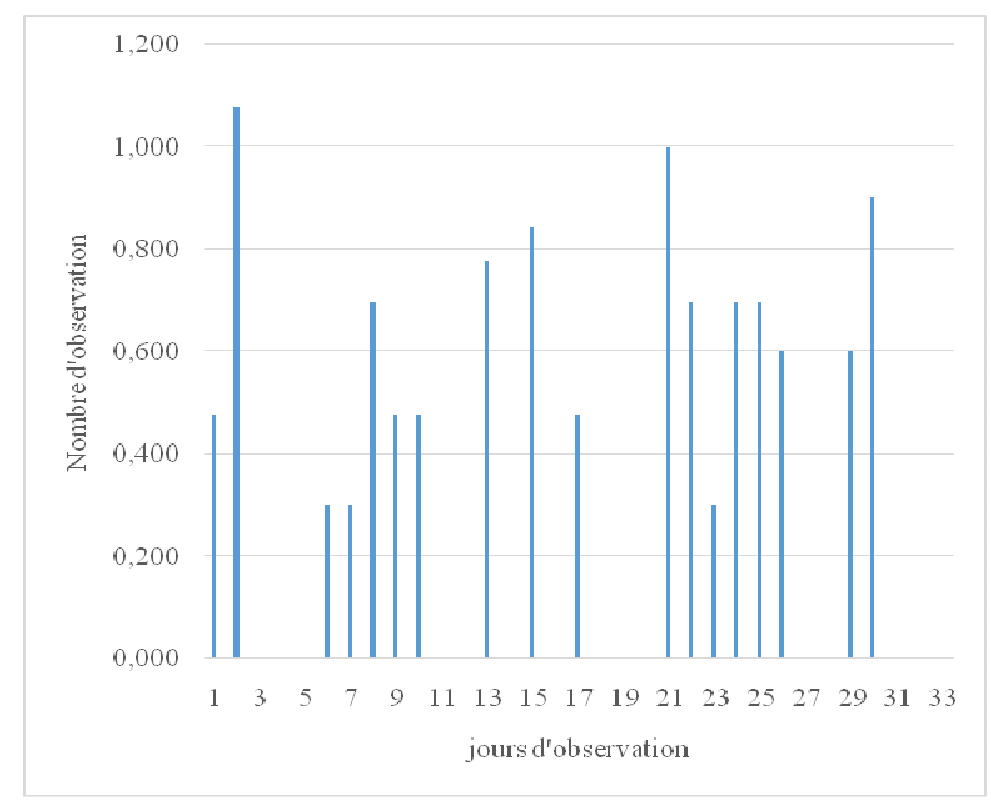

Figure 3 : Répartition des éléphants vus en fonction du temps durant toute la période de l'étude. L'axe des $\mathrm{X}$ correspond au nombre de jours d'observation et l'axe des $\mathrm{Y}$ au nombre d'apparitions des buffles transformé en $\log$ (effectif observé+1). 


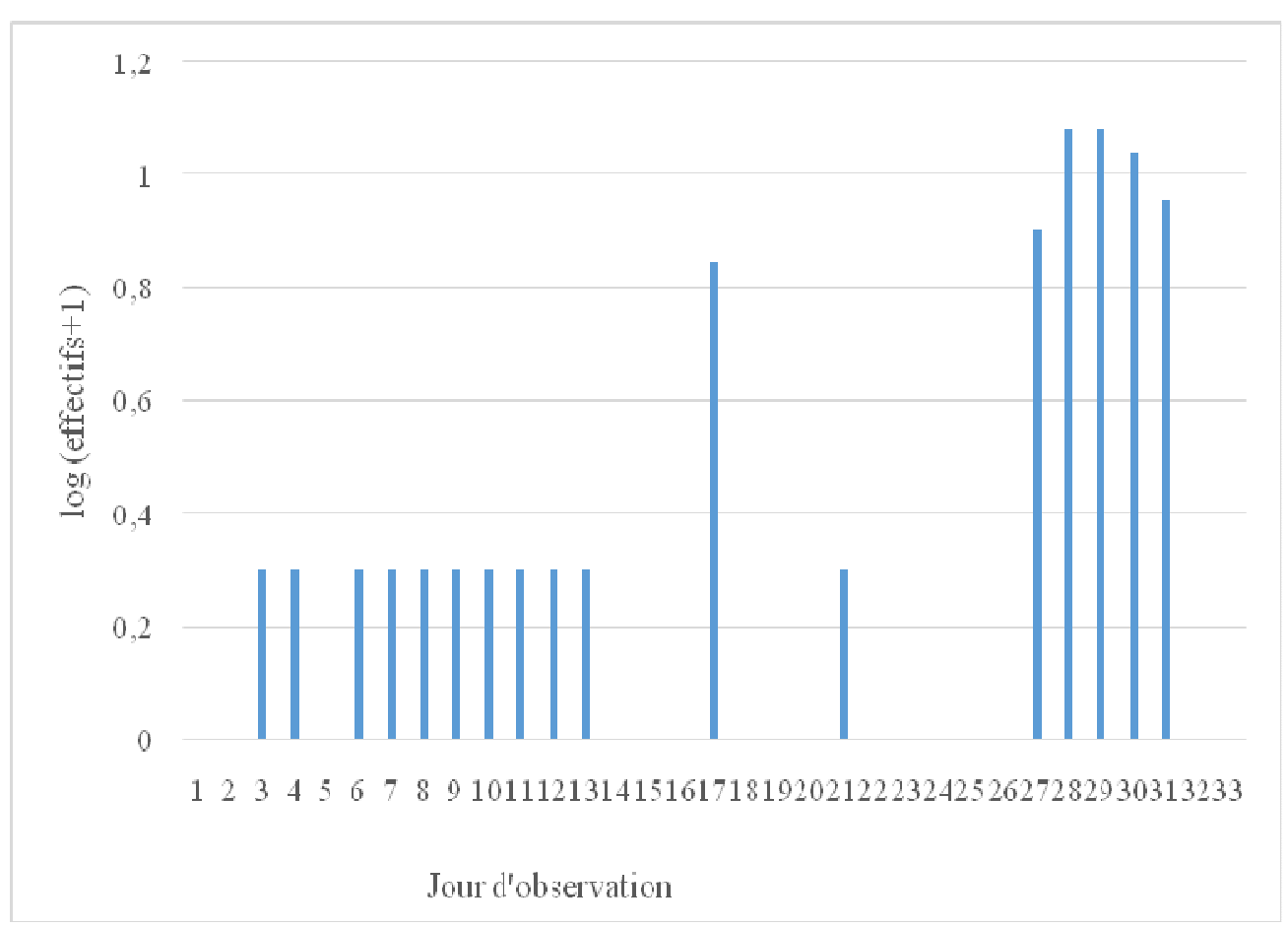

Figure 4: Répartition journalière des buffles observés. L'axe des $\mathrm{X}$ correspond au nombre de jours d'observation et l'axe des Y au nombre d'apparitions des buffles transformé en log (effectif observé+1).

Tableau 1 : Nombre total d'espèces animales observées dans le baï de Momba.

\begin{tabular}{|c|c|c|c|}
\hline Nom commun & Nom scientifique & $\begin{array}{l}\text { Nombre d'individus/ } \\
\text { espèce observées }\end{array}$ & $\begin{array}{c}\text { Fréquence } \\
\text { d'observation }\end{array}$ \\
\hline & & Oiseaux & \\
\hline Palmiste africain & Gypohierax angolensis & 1 & $0,1 \%$ \\
\hline Touraco géant & Corythaeola cristata & 1 & $0,1 \%$ \\
\hline Hirondelle & Hirundo fuliginosa & 7 & $0,7 \%$ \\
\hline Ombrette & Scopus umbretta & 27 & $2,8 \%$ \\
\hline Calaos à casque noir & Ceratogymna atrata & 41 & $4,2 \%$ \\
\hline Aigle pêcheur & Haliaeetus vocifer & 48 & $5,0 \%$ \\
\hline \multirow[t]{2}{*}{ Râle noir } & Limnocorax flavirostra & 402 & $41,5 \%$ \\
\hline & & Mammifères & \\
\hline Loutre & Lutra sp & 2 & $0,2 \%$ \\
\hline Loutre du Congo & Aonyx congicus & 6 & $0,6 \%$ \\
\hline Colobe (singe) & Colobus guereza & 7 & $0,7 \%$ \\
\hline Buffle & Syncerus caffer nanus & 64 & $6,6 \%$ \\
\hline Eléphant & Loxodonta africana cyclotis & 76 & $7,8 \%$ \\
\hline \multirow[t]{2}{*}{ Sitatunga } & Tragelaphus spekei & 286 & $29,5 \%$ \\
\hline & & Reptiles & \\
\hline Varan & Varanus niloticus & 1 & $0,1 \%$ \\
\hline Total & & 969 & \\
\hline
\end{tabular}


Tableau 2 : Identités et classe d'âge des éléphants observés dans le baï de Momba.

\begin{tabular}{cccc}
\hline Date d'apparition & Identités des éléphants observés & Sexe & Classe d'âge \\
\hline $17 / 08 / 2011$ & Zowa & Femelle & $\mathrm{B}(+15$ ans $)$ \\
$17 / 08 / 2011$ & Etsitsi & Femelle & $\mathrm{D}(6$ ans $)$ \\
$17 / 08 / 2011$ & Yakiyene & Femelle & $\mathrm{B}(+15$ ans $)$ \\
$17 / 08 / 2011$ & Pendje & Mâle & $\mathrm{A}(40$ ans $)$ \\
$18 / 08 / 2011$ & Ibacka & Mâle & $\mathrm{A}(+40$ ans $)$ \\
$19 / 08 / 2011$ & Issidié & Femelle & $\mathrm{D}(+6$ ans $)$ \\
$20 / 08 / 2011$ & Mossi & Mâle & $\mathrm{E}(+3$ ans $)$ \\
$21 / 08 / 2011$ & Rosia & Femelle & $\mathrm{A}(+40$ ans $)$ \\
$21 / 08 / 2011$ & Adzibe & Femelle & $\mathrm{C}(10$ ans $)$ \\
$21 / 08 / 2011$ & Moyouwa & Mâle & $\mathrm{A}(+40$ ans $)$ \\
$22 / 08 / 2011$ & Ikani & Femelle & $\mathrm{A}(+40$ ans $)$ \\
$22 / 08 / 2011$ & Dioto & Mâle & $\mathrm{D}(+6$ ans $)$ \\
$25 / 08 / 2011$ & Maniaka & Femelle & $\mathrm{A}(40$ ans $)$ \\
$26 / 08 / 2011$ & Iyama & Mâle & $\mathrm{A}(+40$ ans $)$ \\
$26 / 08 / 2011$ & Bongo & Femelle & $\mathrm{B}(+15$ ans $)$ \\
\hline
\end{tabular}

\section{DISCUSSION}

Cette étude portant sur l'identification de la macrofaune du baï de Momba et la caractérisation de sa dynamique a permis de connaitre et de dresser une liste des différentes espèces de faune fréquentant ce milieu durant la grande saison sèche. Par ailleurs, cette liste pourra servir de référence aux études complémentaires qui seront effectuées ultérieurement afin de connaître l'évolution de ces populations et de déterminer l'importance de ces baïs dans la gestion durable de la biodiversité. De plus, la méthodologie qui a été utilisée au cours de cette étude pourrait être utilisée dans la plupart des baïs présents dans le Gabon et dans le Bassin du Congo.

Au total, 14 espèces animales ont été observées dans le cadre de la présente étude. Cette faible richesse spécifique observée dans cette clairière pourrait s'expliquer par le fait que notre étude a été effectuée seulement durant la grande saison sèche. Par ailleurs, la méthodologie utilisée a consisté à observer les animaux seulement durant la journée. De plus, la collecte des données concernant les grands mammifères, en particulier les éléphants, a été réalisée grâce à des méthodes d'identification mises au point par Turkalo et Fay (1995), Western et al. (1983), Lee et Moss (1995), Reilly (2002), et Morgan et Lee (2003). De ce fait, ces résultats de caractérisation de la grande faune ne sont pas exhaustifs. Cela n'exclut donc pas la présence éventuelle d'autres familles appartenant à la grande faune diurne et /ou nocturne qui n'ont pas pu être observées dans ce travail du fait que les méthodes d'observation des animaux n'ont pas été diversifiées.

La faible richesse des espèces de la grande faune observée au cours de cette étude pourrait s'expliquer la période et à la durée d'étude. En effet, les observations ont été réalisées pendant 33 jours seulement et durant la saison sèche. Par ailleurs, durant cette saison, les mares d'eau du baï sont relativement asséchées. Aussi, les taux de fréquentation de certaines espèces (sitatungas, éléphants et buffles) ont-ils été relativement supérieurs à 5\%. Il ressort de ces résultats que, le baï de Momba pourrait constituer un lieu d'alimentation pour ces animaux et un pôle de concentration animale. Ces résultats sont similaires à ceux réalisés par Magliocca et al. 
(1999), Latour (2003), Temgoua (2006) et Momont (2007). Ces auteurs ont montré que les clairières sont des lieux de rencontre de plusieurs espèces animales.

L'abondance des sitatungas dans le baï de Momba pourrait être liée à leur écologie. En effet, les sitatungas ou guibs d'eau sont des antilopes de forêts marécageuses parfaitement inféodées aux zones humides comme c'est le cas pour le baï de Momba. Par ailleurs, la présence d'une végétation herbeuse constituée par les Graminae pourrait également expliquer leur présence continuelle dans ce milieu. De même, la disponibilité des ressources alimentaires, notamment de la végétation herbeuse dans ce baï attire de nombreuses espèces animales parmi lesquelles les sitatungas (Magliocca et al., 2002). Ces observations corroborent les résultats obtenus par Magliocca et Gautier-Hion (2001) et Magliocca et al. (2002) qui ont montré que les sitatungas sont des espèces qui abondent dans les clairières forestières. De plus, ils ont aussi signifié que les sitatungas seraient des espèces présentant un tropisme positif pour des zones humides et en particulier pour les clairières forestières.

La fréquentation du baï par les buffles semble être hétérogène. En effet, la fréquentation de ces animaux varie selon les jours, soit un buffle observé sur 2 jours. En outre, cette variation pourrait s'expliquer par l'état de la végétation prisée par ces animaux durant la grande saison sèche. Ces résultats sont similaires à ceux obtenus par Latour (2003) qui a montré que les buffles sont moins fréquents dans les baïs en saison sèche. Nos résultats sont en accord avec de nombreux autres travaux qui ont montré que les buffles sont plus fréquemment observés dans les clairières dans les forêts du Bassin du Congo (Magliocca et al., 2002).

La faible fréquentation des éléphants dans le baï pourrait s'expliquer par la période pendant laquelle l'étude a été réalisée c'est-àdire durant la grande saison sèche. Ces résultats corroborent ceux obtenus par Latour
(2003) dans le baï de Langoué au Gabon qui ont signalé que la fréquentation des éléphants diminuait en saison sèche; mais avec une forte activité nocturne (Momont, 2007). Cependant, la présence de ces éléphants dans le baï de Momba pourrait s'expliquer par le fait que ces animaux sont des mégas herbivores et qu'ils entretiendraient une relation étroite avec cet habitat. En effet, les éléphants sont plus précisément considérés comme des ingénieurs de l'écosystème, c'està-dire comme une espèce dont les individus influencent, de façon directe ou indirecte, la disponibilité des ressources en modifiant et/ou en créant des habitats (Owen-Smith 2000). Ainsi, ils peuvent avoir un nombre important d'influences positives (dissémination principale de certaines plantes) sur les écosystèmes. En favorisant la diversité d'habitats et la dissémination des graines des arbres fruitiers dans la forêt équatoriale, ils participeraient au maintien de la structure des communautés végétales et animales (Hawthorne et Parren 2000) puis de certains biotopes comme les clairières forestières.

\section{Conclusion}

Le présent travail pourrait constituer le point de départ d'un programme d'étude sur la dynamique des populations de grands mammifères et autres espèces animales dans les clairières forestières au Gabon. Les résultats obtenus ont permis de montrer que ce milieu est fréquenté par plusieurs espèces de grands mammifères dont les éléphants, les sitatungas et les buffles et d'autres espèces appartenant à divers groupes taxonomiques (mammifères, oiseaux et reptiles). Les informations collectées pourraient être utilisées à court terme et/ou à long terme pour montrer l'importance des clairières forestières et en général des zones humides, dans la gestion durable de la biodiversité végétale et animale du Gabon.

Aussi, il serait nécessaire de mener une étude plus approfondie d'une durée d'un an, sur la fréquentation de ce baï par la macrofaune afin de connaître sa dynamique 
saisonnière et l'éthologie des diverses espèces animales partageant cet espace.

\section{REMERCIEMENTS}

Ce travail a également bénéficié de l'appui institutionnel et logistique de l'Institut de Recherche en Ecologie Tropicale (IRET), du Laboratoire d'Ecologie Vectorielle (LEVIRET), via le projet EU- ACP « Establishment of a Forestry Research network for ACP countries» projet (9 ACP RPR 91 \# 1FORENET). Les auteurs remercient aussi l'Ecole Régionale Post-Universitaire d'Aménagement et de Gestion Intégrée des Forêts et Territoires Tropicaux (ERAIFT) et de la CEEAC/PACEBO, de l'Union Européenne pour leur encadrement. Merci à EMBA YAO et Stéphane ESSASOU BADJI pour leur aide technique sur le terrain. Nos sincères remerciements sont adressés au Docteur MINTSA NGUEMA Rodrigue et Monsieur KOUMBA Aubin dont les remarques, suggestions et corrections ont permis d'améliorer ce manuscrit.

\section{REFERENCES}

Boupoya A. 2010. Flore et Végétation des clairières interforestières sur sol hydromorphe dans le Parc National de l'Ivindo (Nord-est Gabon). Thèse de Doctorat, Université Libre de Bruxelles (France), $246 \mathrm{p}$.

Hawthorne WD, Parren MPG. 2000. How important are forest elephants to the survival of woody plant species in Upper Guinean forest? Journal of Tropical Ecology, 16: 133-150.

Laws RM, Parker JSC, Johnstone RCB.1975. Elephants and their Habitats: The Ecology of Elephants in North Bun Yoro, Uganda. Clarendon Press: Oxford ; 376p.

Lee PC, Moss CJ. 1995. Structural growth in known-age African elephants (Loxodonta africana). Journal of Zoology, London, 236: 29-41.

Magliocca F, Gautier-Hion A. 2001. Les clairières en forêt tropicale : des aires protégées en toute priorité. Canopée, 20: 9.

Magliocca F, Gautier-Hion A. 2016. Minerals contents as the basis for food selection by western lowland gorillas in forest clearings. American Journal of Primatology, In press.

Magliocca F, Querouil S, Gautier-hionA. 1999. Population structure and group composition of Western lowland gorillas in north-western Republic of Congo. American Journal of Primatology, 48(1): 1-14.

Momont L. 2007. Sélection de l'habitat et organisation sociale de l'éléphant de forêt, Loxodonta africana cyclotis (Matschie 1900), au Gabon. Thèse pour obtenir le grade de Docteur du Muséum National d'Histoire Naturelle Discipline : Science de la Vie Spécialité: écologie. 42$128 \mathrm{p}$.

Morgan BJ, Lee PC. 2003. Forest elephant (Loxodonta africana cyclotis) stature in the Réserve de Faune du Petit Loango, Gabon. Journal of Zoology, 259: 337-344.

Nganongo J.B. 1999. Suivi des salines ; Parc national d'Odzala, Congo. Rapports mensuels : Août, septembre, octobre, novembre, décembre.

Nganongo JB. 2000. Suivi des salines ; Parc national d'Odzala, Congo. Rapport mensuel janvier.

Noupa P, Nkongmeneck BA. 2008. Influence des clairières forestières sur la répartition spatiale des grands mammifères dans la forêt dense du Bassin du Congo: cas du Parc National de Boumba-Bek (Sud-est Cameroun). International Journal of Biological and Chemical Sciences, 2(2): 185-195.

Owen-Smith RN. 2000. Megaherbivores. The Influence of Very Large Body Size on Ecology. Cambridge University Press: UK.

Reilly J. 2002. Growth in the Sumatran elephant (Elephas maximus sumatranus) and age estimation based on dung 
diameter. Journal of Zoology, 258: 205-213.

Temgoua LM. 2006. Identification et caractérisation des clairières marécageuses du parc national de l'Ivindo(Gabon) Mémoire pour l'obtention du diplôme de Master en Sciences et Technologies, Agronomie et agro-alimentaire, spécialisation Foresterie Rurale et Tropicale (FRT) ENGREFMontpellier. 19-22p

Turkalo A, Fay JM. 1995. Studying elephants by direct observations: preliminary results from the Dzanga clearing, Central African Republic. Pachyderm, 20: 45-54.

Vande Weghe J-P. 2006. IVINDO et MWAGNA, Eaux noires, forêts vierges et baïs. Ed. WCS : Libreville, Gabon ; 272.

Vanleeuwe H, Cajani S, Gautier-hion A. 1998. Large Mammals at Forest Clearings in the Odzala National Park, Congo. Revue d'Ecologie (Terre Vie), 53: 171180.

WCS. 2008. Langoué baï, Ivindo national Park. Review of the pilot tourism projet, 34p.

Western D, Moss CJ, Georgiadis N. 1983. Age estimation and population age structure of elephants from footprint dimensions. Journal of Wildlife Management, 47(4): 1192-1197.

Zinga-Koumba CR, Acapovi-Yao GL, Mavoungou JF, Kohagne Tongue L, Mbang-Nguema OA, Obame Ondo PK, Shango M. 2013a. Influence de la saison sur l'écodistribution des glossines, tabanides, stomoxes du Baï de Momba Makokou, Gabon. Agronomie Africaine, 25: 149-158.

Zinga-Koumba RC, Bouyer J, Mavoungou JF, Acapovi-Yao GL, Kohagne TL, Mbang Nguema OA, Ondo Obame KP, Mutambwe S. 2013b. Evaluation de la diversité des diptères hématophages dans une clairière marécageuse du Gabon à l'aide des pièges Vavoua et Nzi. Revue d'Elevage et de Médecine Vétérinaire des Pays Tropicaux, 66(3): 91-96.

Zinga-Koumba CR, Mbang-Nguema OA, Kohagne TL, Acapovi-Yao GL, Obame OKP, Mutambwe S, Mavoungou JF. 2014. Contribution à l'évaluation de la diversité des vecteurs biologiques de la Trypanosomose Humaine Africaine et de leur activité journalière dans le Parc National de l'Ivindo (Nord-est Gabon). Journal of Applied Biosciences, 80: 70607070 . 